\title{
Fibre Bragg grating photowriting in microstructured optical fibres for refractive index measurement
}

\author{
M C Phan Huy ${ }^{1}$, G Laffont ${ }^{1}$, Y Frignac ${ }^{1}$, V Dewynter-Marty ${ }^{1}$,

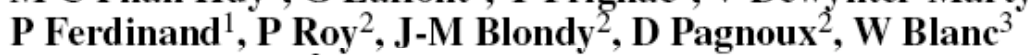 \\ and B Dussardier ${ }^{3}$ \\ ${ }^{1}$ CEA-LIST, Centre d'Etudes de Saclay, 91191 Gif-sur-Yvette Cedex, France \\ ${ }^{2}$ IRCOM/OGI, UMR-CNRS 6615, 123 Avenue Albert Thomas, 87060 Limoges Cedex, \\ France \\ ${ }^{3}$ LPMC/FOA, UMR-CNRS 6622, Université de Nice Sophia-Antipolis, Parc Valrose, \\ 06108 Nice, France \\ E-mail: phanhuy@cea.fr and Guillaume.LAFFONT@cea.fr
}

Received 14 July 2005, in final form 7 March 2006

Published 6 April 2006

\begin{abstract}
We report results relating to fibre Bragg grating photowriting in two kinds of Ge-doped core microstructured optical fibre devoted to sensing applications. A cross-comparison between theoretical and experimental modal field patterns is carried out. We present the first values of the spectral sensitivity of a Bragg grating in relation to the refractive index of calibrated oils inserted into the holes.
\end{abstract}

Keywords: microstructured optical fibre, fibre Bragg grating, sensor, refractive index, modelling

(Some figures in this article are in colour only in the electronic version)

\section{Introduction}

Since the beginning of the $1990 \mathrm{~s}$, photonic crystal fibres have been an increasing topic of interest in R\&D laboratories because of their large potentialities in several application fields, such as nonlinear effects [1,2], dispersion compensation $[3,4]$, high power propagation [5] and sensors [6]. Classically, we can distinguish photonic crystal fibres guiding light by the photonic bandgap effect [7] and microstructured optical fibres (MOFs) guiding light by total internal reflection.

For the latter, several processes have been proposed to photowrite fibre Bragg gratings (FBG), depending on the composition of the fibre. For pure silica MOF, Groothoff et al [8] use a two-photon absorption process at $193 \mathrm{~nm}$ to obtain FBG. Kakarantzas et al [9] write long-period gratings (LPGs) using a $\mathrm{CO}_{2}$ laser. In this case, holes of the MOF are periodically collapsed by heat treatment with the laser. Humbert et al [10] choose to use an electric arc technique to realize LPGs. For MOF with a doped core, Canning et al [11] use a two-photon absorption process at $193 \mathrm{~nm}$ to realize gratings in $\mathrm{Er}^{3+}$-doped aluminosilicate core photonic crystal fibre in order to develop a photonic crystal fibre laser. For Ge-doped photosensitive fibres, Eggleton et al [12] write both FBGs and LPGs using a phase mask setup with a frequency-doubled excimer-pumped dye laser. Thus, several photowriting techniques exist. According to the desired application, we have to determine the kind of fibre and the photowriting process adapted to it.

One approach for refractometry applications using conventional fibres is to write LPGs [13] or tilted fibre Bragg grating (TFBGs) [14], because they are sensitive to the refractive index of the medium surrounding the fibre cladding. Another possibility is to use FBGs [15] or LPGs [16] in D-shape optical fibre with sensitivity enhancement through cladding etching. The main interest of $\mathrm{MOF}$, in comparison with conventional fibres, is that the interaction between the guided optical electromagnetic field and the medium inserted into the holes is stronger. Increasing this interaction can be achieved by a convenient design of hole geometry and layout (MOF design).

In this paper, we present the design and the manufacturing of microstructured optical fibres with a Ge-doped core. 
Moreover, FBGs are photowritten in these fibres to spectrally encode and remotely detect the requested information (any change of the refractive index). Experimental results of modal imaging are compared to modelling results obtained using a home-made simulation tool based on the localized functions method. We have then inserted calibrated liquid oils into the channels of the manufactured fibre to determine the sensitivity of FBGs to refractive index with the prospect of refractometry applications.

\section{Simulation method}

In order to define a convenient design of microstructured fibres for FBG sensing applications, we have implemented a simulation tool based on a scalar version of the localized functions method (LFM). First proposed by Mogilevtsev et al [17], this simulation tool has been significantly developed and modified by Monro et al [18]. This method consists in projecting the modal electric field on a basis of localized functions, Hermito-Gaussian (HG) functions. In our version of this simulation tool, the decomposition of the index profile is adapted to consider the Ge-doped core [19]. In this way, the index profile is described by the sum of sinusoidal functions (describing the cladding) and HG functions (describing the core). This tool allows us to accurately model the guided mode characteristics (effective index $n_{\text {eff, }}$ effective area $A_{\text {eff }}$ and modal field patterns) taking into account the air-hole distributions and the refractive index of the substance injected into the holes [19].

In a first step, this simulation tool is validated by comparing our results to those obtained by Peyrilloux et al [20] using the finite element method (FEM), for an ideal singlemode MOF with a four-ring triangular pattern, $2 \mu \mathrm{m}$ pitch and $0.5 \mu \mathrm{m}$ hole diameter. The difference between LFM-

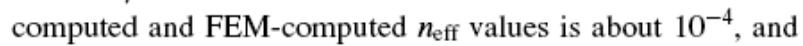
for $A_{\text {eff }}$ values the difference is about $0.2 \mu \mathrm{m}^{2}$. Then, in a second step, we validate the simulation tool by comparing our simulation results to those obtained by Kerbage et al [21]. We analyse a multimode Ge-doped core MOF comprising six large air holes. This fibre is described by Kerbage et al and modelled by the beam propagation method (BPM) [21]. In figure 1, we report the modal field patterns for the first five guided modes given by the LFM [19] and the BPM, as well as experimentally observed modes. For fundamental and higher order guided modes, LFM-computed and BPM-computed $n_{\text {eff }}$ values (difference below $2 \times 10^{-4}$ ) are in very good agreement with those given by Kerbage et al.

The simulation tool has been validated through comparison with published results obtained with the FEM and BPM. Now, we want to validate this simulation tool by comparison to measured results (e.g. modal field patterns) obtained with fibres and gratings that we have designed and manufactured.

\section{Design and manufacturing of microstructured fibres and FBG photowriting}

MOF manufacturing consists of realizing a preform. The preform is a stack of silica capillaries and rods (a few millimetres long). For a Ge-doped core MOF, we replace

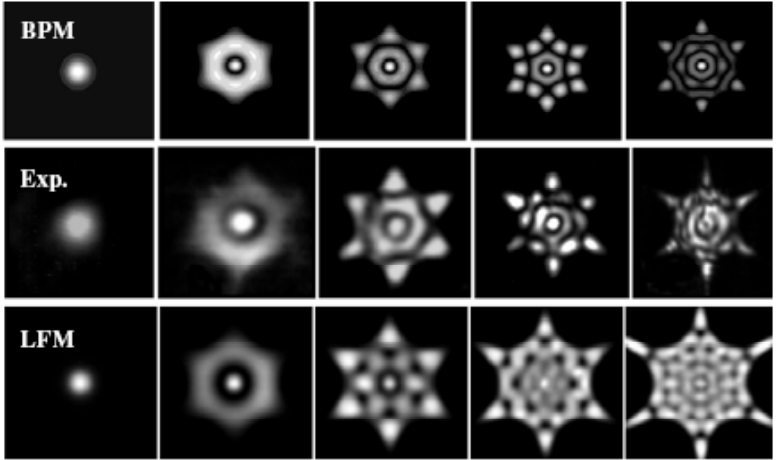

Figure 1. Modal field patterns of the first five guided modes calculated by the BPM (top line) [21], the LFM (bottom line) and experimentally observed (middle line) [21].

the central silica rod with a Ge-doped silica rod of equal diameter. The Ge-doped preform is manufactured using the MCVD technique. In order to reduce the diameter of such a preform, two successive steps are necessary to reach the required diameter. A mechanical machining is used to reduce the diameter from $12 \mathrm{~mm}$ to $2 \mathrm{~mm}$, followed by a chemical attack (HF acid) to get a diameter close to $1 \mathrm{~mm}$. We then use a conventional stack and draw process to make the fibres. The first manufactured fibre presents one ring of six air holes of diameter $d \sim 15 \mu \mathrm{m}$ and pitch $\Lambda \sim$ $15.8 \mu \mathrm{m}$, surrounding a Ge-doped core of $11 \mu \mathrm{m}$ that is noncircular due to inflation of holes (see figure $2(a)$ ); the index contrast ( $n_{\text {core }}-n_{\text {cladding }}$ ) is about $8 \times 10^{-3}$. Such fibre is expected to be multimode $(d / \Lambda=0.95)$. The second fibre has a two-ring triangular pattern of respectively 6 and 12 air holes with diameter $d \sim 3.1 \mu \mathrm{m}$ and pitch $\Lambda \sim 6.7 \mu \mathrm{m}$, surrounding a Ge-doped core of $5 \mu \mathrm{m}$ (slightly decentred) (see figure $2(b)$ ). The fibre obtained is slightly multimode (three modes identified) in the $1.5 \mu \mathrm{m}$ spectral window.

The FBG photowriting is performed using a Lloyd mirror interferometer setup which includes a $\mathrm{CW}$ frequency-doubled argon laser emitting at $244 \mathrm{~nm}$. Prior to the photowriting step, microstructured optical fibres are hydrogen loaded at $160 \mathrm{bar} / 25^{\circ} \mathrm{C}$ for one week to enhance the photosensitivity of the Ge-doped core (a FBG with a reflectivity of $75-80 \%$ is photowritten in $15 \mathrm{~min}$, UV power $=80 \mathrm{~mW}$ ). Figure 3 illustrates the transmission spectrum of FBGs written in the core of the two microstructured fibres. $6 \mathrm{~mm}$ long FBG are photowritten at $1560.5 \mathrm{~nm}$ and at $1555.5 \mathrm{~nm}$, respectively, for the six-hole and the two-ring triangular MOF. Gratings reflectivities reach $80 \%$ in both cases and the grating FWHM is roughly equal to $300-350 \mathrm{pm}$. The FBG allows us to spectrally separate modes of a given MOF. For the six-hole MOF, the Bragg resonance as well as other higher order resonances (see figure $3(a)$ ) can be distinguished in the transmission spectrum of the FBG.

\section{Modal imaging: cross-comparison between simulation and experimental results}

The modal imaging setup (figure 4) includes an InGaAs CCD camera $(316 \times 252$ pixels, pixels size of $30 \mu \mathrm{m} \times 30 \mu \mathrm{m})$, an IR tunable laser source, two $\times 40$ microscope objectives and a 

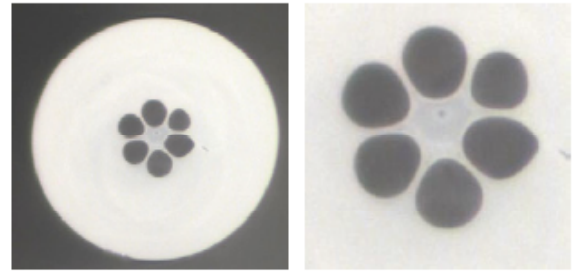

(a)
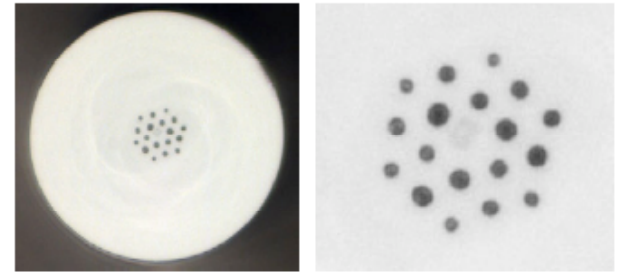

(b)

Figure 2. Microscope image of $(a)$ the six holes and $(b)$ the two-ring triangular photosensitive MOF.

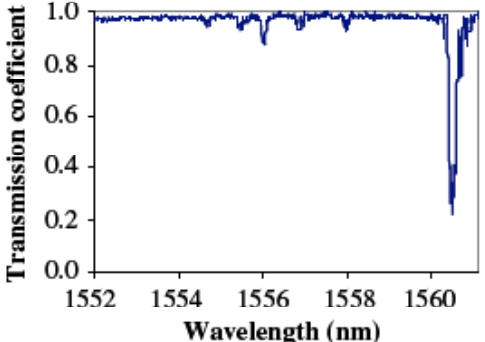

(a)

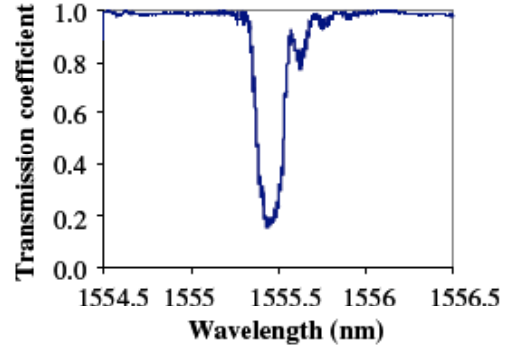

(b)

Figure 3. Transmission spectrum of FBG photowritten in $(a)$ the six-hole and $(b)$ the two-ring triangular MOF.

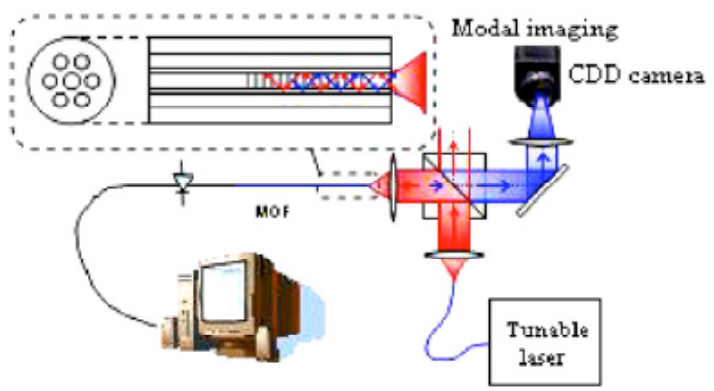

Figure 4. Near-IR modal imaging setup.

beam splitter $(50 / 50)$. The fibre is cleaved close to the FBG in order to increase the signal-to-noise ratio, so guided modes with loss can be clearly apparent. Our acquisition of modal field patterns is performed in two steps. Firstly, we acquire the FBG's spectral response in transmission. That spectrum yields us the resonance wavelength of each mode. Then, we successively tune the laser to any of the resonance wavelengths and get the image of each reflected mode on the camera.

Furthermore, high quality cross section images of the two manufactured fibres have been acquired thanks to an optical microscope. These images are used as input parameters for the simulation tool which provides modal field patterns that we can directly compare with those observed experimentally. The fact that we use a periodic function to describe the cladding structure requires us to determine a calculation window to have a finite cladding structures (the window size is the size in $\mu \mathrm{m}$ of the image used as input parameter). Calculation windows are squares measuring $46 \mu \mathrm{m}$ and $35 \mu \mathrm{m}$ on a side respectively for the six-hole and the two-ring triangular fibres.

For the two-ring triangular fibre, the numerically simulated mode pattern corresponds to the experimental results (see figure 5(a)). We identify field patterns for the first three resonances corresponding to the first three modes, including the fundamental one. The field patterns of the two resonances at low wavelengths are not clearly apparent: they probably correspond to leaky modes. Leaky modes are exclusively observed as dips in the FBG spectral response observed in transmission, while guided modes are also apparent in reflection as resonance peaks (see figure 5(a)). For the six-hole fibre, we can notice some differences between the experimental and theoretical modes: two- and three-lobe modes observed experimentally do not appear in the simulation results; on the contrary one mode made of two lobes obtained by simulation is not observed (see figure $5(b)$ ). We suppose that these differences are due to an inaccuracy in the definition of the doped core shape fed to the simulation software. Thanks to the index contrast on the microscope image of the six-hole fiber we can determine the shape of the Ge-doped core and use it for the simulation with the LFM and FEM, but maybe the definition of the doped core shape is not accurate enough to explain the differences between modes obtained experimentally and by simulation.

Because the fibre structure is asymmetric, we can suppose that polarization effects occur: birefringence may for instance induce a splitting of the spectral resonances. A full-vectorial modelling using the finite element method (FEM) is performed and the results are compared to those obtained by LFM, using the same six-hole fibre microscope picture in both cases.

In figure $6(a)$, we compare the $n_{\text {eff }}$ values obtained respectively with the LFM and FEM methods. Due to the birefringence of the microstructured fibre, the symmetry between two degenerated spatial modes is broken. Therefore, using the full-vectorial modelling, we retrieve two values of $n_{\text {eff }}$ instead of a single one with the LFM method. This difference ( $\Delta n_{\text {eff }}$ ) between the two FEM-computed $n_{\text {eff }}$ values of spatial modes (see figure $6(a)$ ) varies from $6 \times 10^{-6}$ to $3.2 \times 10^{-5}$ and it is represented by a line with diamonds in figure 6(a). In wavelength, the difference is about $3 \mathrm{pm}$ to $18 \mathrm{pm}$ (represented 


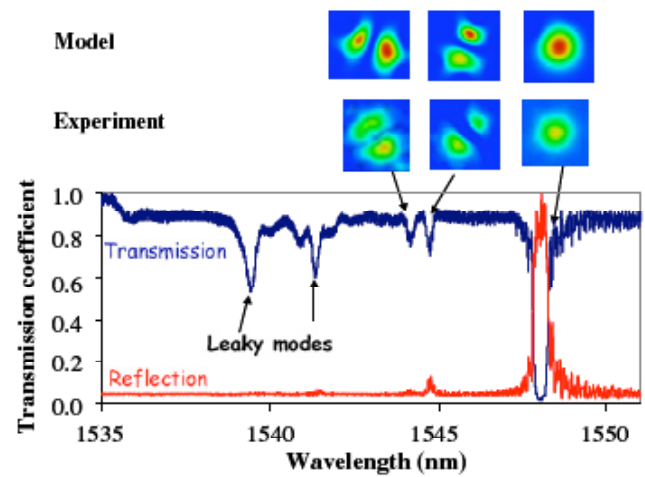

(a)

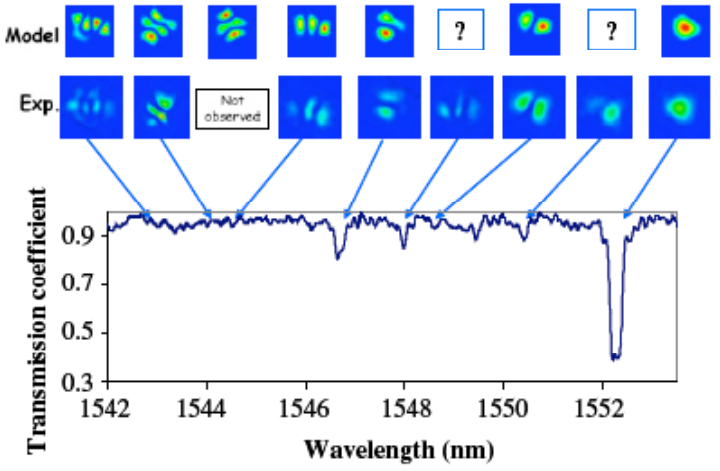

(b)

Figure 5. Experimental FBG transmission spectrum with corresponding experimental and simulated modal field pattern for $(a)$ the two-ring triangular MOF and $(b)$ the six-hole fibre.

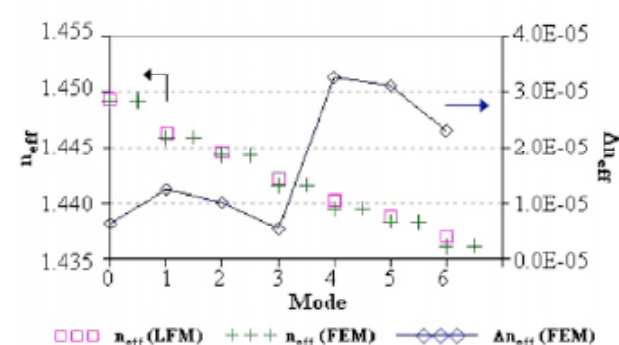

(a)

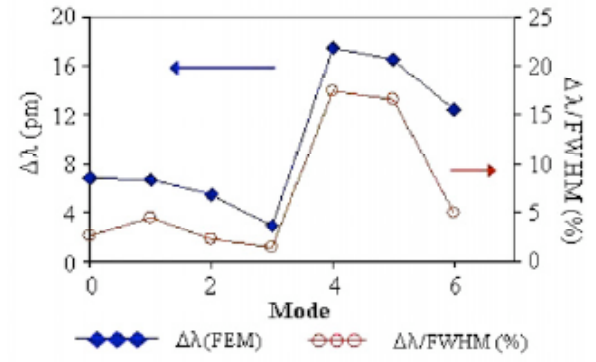

(b)

Figure 6. (a) Effective indices of the modes obtained respectively with the LFM and FEM; the diamond line represents the difference in effective index between two polarizations for the six-hole fibre (cf figure 2(a)). $(b)$ Difference in wavelength between two modes coming from the splitting in two of the spectral resonances; circles represent the ratio of the variation in wavelength to the FWHM (of each resonance) as a percentage.

LFM

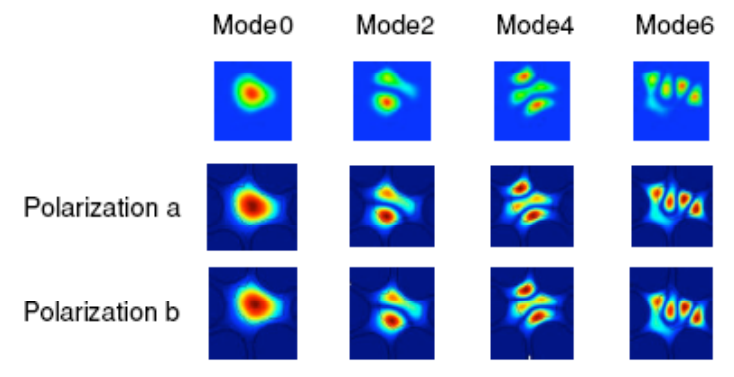

Figure 7. Field norms obtained with the LFM and FEM of mode 0, mode 2 , mode 4 and mode 6.

by diamonds in figure $6(b)$ ). These values are lower than the FWHM of each resonance, which is about $300 \mathrm{pm}$. In figure $6(b)$, we present (by circles) the ratio of $\Delta \lambda$ to the FWHM (of each resonance). $\Delta \lambda$ corresponds to the splitting in two of the resonant wavelength due to the birefringence of the fibre. Ratio values are less than $20 \%$, showing that it is not possible to see and separate experimentally the two resonances corresponding to two polarization states. Now, if we compare LFM- and FEM-computed $n_{\text {eff, }}$, we notice that their absolute values are similar, the maximum difference being about $10^{-3}$ (see figure 6(a)). The norms of the electric field obtained with the FEM and LFM are also similar. The electric field norms between two modes coming from the splitting in two of resonant modes are identical (see figure 7).

\section{Influence of the hole's refractive index on the FBG's spectral response}

To determine the spectral sensitivity of FBGs photowritten in the two manufactured fibres according to the refractive index of a substance inserted into the holes, several liquids of calibrated refractive index (Cargille's oils) are easily introduced by capillarity into the fibre sections (Cargille's oils are not very viscous, about $v \sim 15 \mathrm{cSt}$ ). For each refractive index oil, a new fibre section and thus a new FBG with the same spectral characteristics (wavelength, FWHM and reflectivity) is used to avoid measurement errors due to previous traces of liquid. The refractive index $n_{1}$ of the liquids we have used varies from 1.2960 to 1.4510 (at $1550 \mathrm{~nm}$ and $25^{\circ} \mathrm{C}$ ). The insertion of a fluid changes the cladding refractive index and modifies strongly the interaction between the evanescent field of the guided mode and the medium in the holes. The effective index of the guided mode increases together with the refractive index of the holes. So, when a given liquid reaches the grating, the Bragg resonance experiences a red shift, as depicted in figure 8 .

For the six-hole fibre, we notice that experimental and simulated curves are not in full agreement (see figure 8(a)). We explain this difference by taking into account a circular core for the model and not the real core shape (non-circular core) and by the non-homogeneity of the refractive index profile between different fibre sections. 


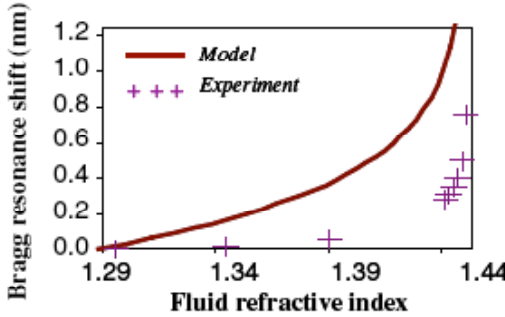

(a)

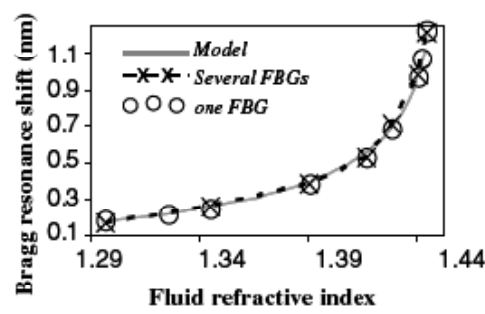

(b)

Figure 8. Bragg resonance shift corresponding to the liquid refractive index in (a) the six-hole and $(b)$ the two-ring triangular MOF.

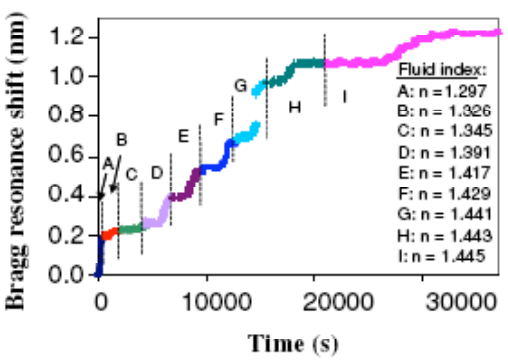

Figure 9. Bragg resonance shift versus time when extra refractive index oils are successively inserted into the holes of the two-ring triangular MOF.

For the two-ring triangular fibre, a first experiment consists of inserting several liquids in different sections of the fibre and thus different FBGs (so one liquid for one FBG, represented by crosses in figure $8(b))$. Experimental and simulated results are in very good agreement, the difference being about $34 \mathrm{pm}$ at most. In a second experiment, we repeat this experiment with a single section and hence a single FBG. We successively insert fluids of different refractive index, without removing the previous liquid (see figure 9) and observe the spectral shift of the Bragg wavelength resonance (represented by circles in figure $8(b)$ ). We notice that the three curves (model, measurement with several FBGs, measurement with one FBG) are in very good agreement, the difference between simulated and experimental results being close to $34 \mathrm{pm}$.

The index profile is homogeneous enough along the fibre and previous liquid traces are low enough not to influence our measurements (in the limit of resolution of our measurement system).

In table 1 , we report the expected refractive index resolution for different operating point values, with the assumption that the measurement system can detect a spectral variation as low as $1 \mathrm{pm}[22,23]$. This resolution has been obtained with FBG measurements in conventional optical fibres and further work will check experimentally the expected refractive index resolution and hence involve a full metrological study of this new class of sensor. Within the range $1.3-1.39$ for the liquid refractive index $n_{1}$, the Bragg resonance shift is relatively weak (about $60 \mathrm{pm}$ and $200 \mathrm{pm}$ respectively for the six-hole fibre and the two-ring triangular fibre, for a variation in liquid refractive index of 0.09 ri.u. (refractive index unit)). The resolution reached is $\Delta n$ six-hole $\sim 8 \times$ $10^{-4}$ r.i.u. and $\Delta n_{\text {two-ring }} \sim 4 \times 10^{-4}$ r.i.u. When the refractive index $n_{\mathrm{l}}$ approaches the effective index of the fundamental
Table 1. Refractive index resolution of fluid inserted into the fibre holes (based on a 1 pm spectral resolution [22]).

\begin{tabular}{llrlrr}
\hline \multirow{2}{*}{$\begin{array}{l}\Delta \lambda=1 \mathrm{pm} \\
\text { Index of the }\end{array}$} & \multicolumn{3}{c}{ Detectable index variation $(\Delta n)$} \\
\cline { 2 - 3 } $\begin{array}{l}\text { fluid at } \\
1550 \mathrm{~nm}\end{array}$ & \multicolumn{2}{c}{ Two-ring triangular MOF } & & \multicolumn{2}{c}{ Six-hole MOF } \\
\cline { 2 - 3 } \cline { 5 - 6 } & Model & Experiment & & Model & \multicolumn{1}{c}{ Experiment } \\
\hline$\sim 1.33$ & $5.7 \times 10^{-4}$ & $7 \times 10^{-4}$ & & $3.2 \times 10^{-4}$ & $4 \times 10^{-3}$ \\
$\sim 1.40-1.41$ & $1.5 \times 10^{-4}$ & $1.8 \times 10^{-4}$ & & $1.4 \times 10^{-4}$ & $2.1 \times 10^{-4}$ \\
$\sim 1.44$ & $2.4 \times 10^{-5}$ & $2 \times 10^{-5}$ & & $3.1 \times 10^{-5}$ & $7 \times 10^{-5}$ \\
$\sim 1.45$ & & & $3.4 \times 10^{-6}$ & $6.8 \times 10^{-6}$ \\
\hline
\end{tabular}

mode, the Bragg resonance shift is the most important, corresponding to the highest refractive index resolution ( $\Delta n_{\text {six-hole }} \sim 7 \times 10^{-6}$ r.i.u. for $n_{1} \sim 1.45$ and $\Delta n_{\text {two-ring }} \sim 2 \times$ $10^{-5}$ r.i.u for $n_{1} \sim 1.44$ ).

We point out that the doped core position influences the resolution index. The decentring of the $\mathrm{Ge}$-doped core reduces the interaction between the optical electromagnetic field and liquids inserted into the holes, leading to losses in index resolution. So, with a better accuracy in fibre manufacturing, the index resolution will be improved.

\section{Conclusion}

Two microstructured optical fibres have been designed and manufactured, and fibre Bragg gratings have been photowritten in their Ge-doped cores. Modal imaging is realized and the results are compared to simulated results from the LFM (scalar version) and FEM (vectorial modelling tool). For the sixhole fibre, the full-vectorial modelling with the FEM allows us to take into account the birefringent nature of the fibre (due to its asymmetric structure). We show that it is not possible to experimentally observe the splitting in two of the resonant wavelengths due to birefringence. For these two fibres, the spectral sensitivity of FBG to refractive index of calibrated oils inserted into the holes (ranging from 1.29 to 1.45) has been measured. These results have been compared to simulation results obtained using the localized functions method. Regarding sensing applications, with the assumption that the analysis system has a spectral resolution of $1 \mathrm{pm}$, the refractive index resolution is expected to be equal to $7 \times 10^{-4}$ r.i.u. and $4 \times 10^{-3}$ r.i.u., respectively for the two-ring triangular and the six-hole MOF, for a value of refractive index close to that of water $\left(n_{1} \sim 1.33\right)$. For a value of the refractive index $\left(n_{1} \sim 1.44\right.$ for the two-ring triangular fibre and $n_{1} \sim 1.45$ for the six-hole fibre, at $1550 \mathrm{~nm}$ ) close to the effective index of the guided mode, we expect the 
refractive index resolution to reach $2 \times 10^{-5}$ r.i.u. and $7 \times$ $10^{-6}$ r.i.u., respectively for the two-ring triangular and the six-hole MOF. To enhance the resolution, we can increase either the sensitivity of the FBG to the refractive index of the oils or the resolution of the measurement system. Sensitivity improvement requires the optimization of the refractive index profile of the microstructured fibre, in order to enhance the interaction between the optical electromagnetic field and the sensitive products inserted into the fibre holes. The simulation tool validated by the modal analysis will be used to achieve this goal.

\section{Acknowledgments}

This research is co-funded by the French Ministry of Research-ACI Nouvelles Méthodologies Analytiques et Capteurs 2003 and by the INRS (Institut National de Recherche et de Sécurité). We thank reviewers for their constructive comments on the article.

\section{References}

[1] Broderick N G R, Monro T M, Bennet P J and Richardson D J 1999 Non linearity in holey optical fibers: measurement and future opportunities Opt. Lett. 24 1395-7

[2] Ju H L, Belardi W, Monro T M and Richardson D J 2003 Holey fiber based nonlinear optical devices for telecommunications Proc. 29th Conf. on Lasers and Electro-Optics/Quantum Electronics and Laser Science (Baltimore)

[3] Andrés P, Ferrando A, Silvestre E, Miret J J and Andrés M V 2002 Dispersion and polarization properties in photonic crystal fibers Proc. 4th Int. Conf. on Transparent Optical Networks

[4] Bjarklev A, Broeng J, Barkou S and Dridi K 1998 Dispersion properties of photonic crystal fiber 24th European Conf. on Optical Communication (Madrid)

[5] Wadsworth W J, Percival R M, Bouwmans G, Knight J C and Russell P S J 2003 High power air-clad photonic crystal fiber laser Opt. Express 11 48-53

[6] Pagnoux D et al 2005 Microstructured fibers for sensing applications Proc. 17th Int. Conf. on Optical Fibre Sensors (Bruges) invited paper

[7] Knight J C, Birks T A, Russel P S J and Atkin D M 1996 All-silica single-mode optical fiber with photonic crystal cladding Opt. Lett. 21 1547-9

[8] Groothoff N, Canning J, Buckley E, Lyttikainen K and Zagari J 2003 Bragg gratings in air-silica structured fibers Opt. Lett. $28233-5$
[9] Kakarantzas G, Birks T A and Russel P S J 2002 Structural long-period gratings in photonic crystal fibers Opt. Lett. 27 1013-5

[10] Humbert G, Malki A, Février S, Roy P and Pagnoux D 2003 Electric arc-induced long-period gratings in Ge-free air-silica microstructure fibres Electron. Lett. 39 349-50

[11] Canning J, Groothoff N, Buckley E, Ryan T, Lyttikainen K and Digweed J 2003 All-fibre photonic crystal distributed Bragg reflector (PC-DBR) fibre laser Opt. Express 11 1995-2000

[12] Eggleton B J, Westbrook P S, Windeler R S, Spalter S and Strasser T A 1999 Grating resonances in air-silica microstructured optical fibers Opt. Lett. 24 1460-2

[13] James S W and Tatam R P 2003 Optical fibre long-period grating sensors: characteristics and application (review article) Meas. Sci. Technol. 14 R49-R61

[14] Laffont G and Ferdinand P 2001 Tilted short-period fiber-Bragg-grating-induced coupling to cladding modes for accurate refractometry Meas. Sci. Technol. 12 765-70

[15] Zhou K, Chen X, Zhang L and Bennion I 2005 Optical chemsensors based on etched fibre Bragg gratings in D-shape and multimode fibres Proc. 17th Int. Conf. on Optical Fibre Sensors (Bruges) pp 158-61

[16] Chen X, Zhou K, Zhang L and Bennion I 2004 Optical chemsensors utilizing long-period fiber gratings UV-inscribed in D-fiber with enhanced sensitivity through cladding etching IEEE Photonics Technol. Lett. $161352-4$

[17] Mogilevtsev D, Birks T A and Russel P S J 1998 Group-velocity dispersion in photonic crystal fibers $O p t$. Lett. 23 1662-4

[18] Monro T M, Richardson D J, Broderick N G R and Bennett P J 1999 Holey optical fibers: an efficient modal model IEEE $J$. Lightwave Technol. 17 1093-101

[19] Parmentier R, Phan Huy M C, Laffont G, Dewynter-Marty V, Ferdinand P, Roy P, Blondy J M, Pagnoux D and Dussardier B 2004 Cross comparison between theoretical and experimental modal field patterns in a doped-core microstructured fiber Proc. POWAG Conf. (Bath)

[20] Peyrilloux A, Février S, Marcou J, Pagnoux D and Sansonetti P 2002 Comparison between the finite element method, the localized function method and a novel equivalent average index method for modeling photonic crystal fibers J. Opt. A: Pure Appl. Opt. 4 257-62

[21] Kerbage C E, Eggleton B J, Westbrook P S and Windeler R S 2000 Experimental and scalar beam propagation analysis of an air-silica microstructured fiber Opt. Express 7 113-22

[22] Laffont G, Roussel N, Maurin L, Boussoir J, Clogenson B, Auger L, Magne S and Ferdinand P 2005 Wavelength tunable fiber ring laser for high-speed interrogation of fiber Bragg grating sensors Proc. 17th Int. Conf. on Optical Fibre Sensors (Bruges)

[23] Dyer S D, Williams P A, Espejo R J, Kofler J D and Etzel S M 2005 Fundamental limits in fiber Bragg grating peak wavelength measurements Proc. 17th Int. Conf. on Optical Fibre Sensors (Bruges) invited paper 